\title{
FINITE SIZE SCALING ANALYSIS OF SU(2) LATTICE GAUGE THEORY IN (3 + 1) DIMENSIONS*
}

\author{
J. ENGELS, J. FINGBERG and M. WEBER \\ Fakultät für Physik, Universität Bielefeld, D-4800 Bielefeld, Fed. Rep. Germany
}

Received 24 August 1989

\begin{abstract}
We have calculated the order parameter, the susceptibility and the normalized fourth cumulant $g_{r}$ with high precision on $N_{\sigma}^{3} \times 4$ lattices $\left(N_{o}=8,12,18\right.$ and 26) for $\mathrm{SU}(2)$ gauge theory at finite temperature. The finite size scaling analysis of these quantities confirms that the critical exponents of $S U(2)$ gauge theory are the same as those of the three-dimensional Ising model, the infinite volume value for the critical coupling is $4 / g_{\mathrm{c}}^{2}\left(N_{\tau}=4\right)=2.2985 \pm 0.0006$. With direct scaling fits we determine $\beta / \nu$ and $\gamma / \nu$ and find excellent agreement with the hyperscaling relation.
\end{abstract}

\section{Introduction}

In full QCD Monte Carlo simulations with intermediate bare quark masses (see, e.g., the review in ref. [1]) and even in pure SU(3) lattice gauge theory [2] the order of the finite temperature transition is still undetermined or at least in question. This uncertainty is not only due to the small lattices in use for full QCD, but also to the criteria and methods with which a first or higher order transition is identified on finite lattices. To improve the situation it is then of importance to study the behavior of thermodynamic quantities near a clear second order deconfinement transition as a function of lattice size. Pure SU(2) lattice gauge theory contains already essential features of full QCD and shows such a second order phase transition, which can - in reasonable computer time - be studied with high statistics even on relatively large lattices.

To be more detailed, we want to apply finite size scaling techniques to $\mathrm{SU}(2)$ to check the universality hypothesis of Svetitsky and Yaffe [3]. That conjecture implies that SU(2) lattice gauge theory should have the same critical exponents as the three-dimensional Ising model. In addition, the method of finite size scaling allows for an accurate determination of the critical point in the thermodynamic limit and a check on hyperscaling relations.

* Work supported by the Deutsche Forschungsgemeinschaft under research grant En 164/2. 
This paper is organized as follows. In sect. 2 we discuss the thermodynamic quantities with critical behaviour and the data which we obtained for them. A review of that part of finite size scaling theory, which is relevant for our problem is given in sect. 3. In sect. 4 we present applications: the determination of the critical point, the check of the universality hypothesis and direct scaling fits. We close with a summary in sect. 5 .

\section{Thermodynamic quantities with critical behaviour}

We simulated $\mathrm{SU}(2)$ gauge theory on $N_{\mathrm{o}}^{3} \times N_{\tau}$ lattices for the standard Wilson action

$$
S(U)=\left(4 / g^{2}\right) \sum_{\mathrm{P}}\left(1-\frac{1}{2} \operatorname{Tr} U_{\mathrm{P}}\right),
$$

where $U_{\mathrm{p}}$ is the product of link operators around a plaquette. The number of lattice points in space (time) direction $N_{\sigma(\tau)}$ and the lattice spacing $a$ fix the volume and temperature

$$
V=\left(N_{\sigma} a\right)^{3}, \quad T=1 / N_{\tau} a .
$$

For a given coupling constant $g^{2}$ the lattice spacing may be expressed in units of the lattice scale parameter $\Lambda_{\mathrm{L}}$ by assuming the asymptotic scaling relation for $a\left(g^{2}\right)$

$$
a\left(g^{2}\right) \Lambda_{\mathrm{L}}=\exp \left\{-\frac{12 \pi^{2}}{11 g^{2}}+\frac{51}{121} \ln \left(\frac{24 \pi^{2}}{11 g^{2}}\right)\right\} .
$$

On an infinite volume lattice the order parameter for the deconfinement transition is the expectation value of the Polyakov loop

$$
L(\boldsymbol{x})=\frac{1}{2} \operatorname{Tr} \prod_{\tau=1}^{N_{\tau}} U_{\tau, \boldsymbol{x} ; 0},
$$

or else, that of its lattice average

$$
L=1 / N_{\sigma}^{3} \sum_{x} L(x),
$$

where $U_{x ; 0}$ are the SU(2) link matrices at four-position $x$ in time direction. In the neighbourhood of the critical temperature $T_{\mathrm{c}}$ one expects for $N_{\sigma} \rightarrow \infty$

$$
\langle L\rangle \sim\left(T-T_{\mathrm{c}}\right)^{\beta}, \text { for } T \rightarrow T_{\mathrm{c}}^{+} .
$$

The behaviour near $T_{\mathrm{c}}$ of the susceptibility $\chi$, the correlation length $\xi$ and the 
specific heat $C_{\mathrm{v}}$ is determined by other critical exponents. For $N_{\sigma} \rightarrow \infty$ one expects

$$
\begin{gathered}
\chi \sim\left|T-T_{\mathrm{c}}\right|^{-\gamma}, \text { for } T \rightarrow T_{\mathrm{c}}, \\
\xi \sim\left|T-T_{\mathrm{c}}\right|^{-\nu}, \text { for } T \rightarrow T_{\mathrm{c}}, \\
C_{\mathrm{v}} \sim\left|T-T_{\mathrm{c}}\right|^{-\alpha}, \text { for } T \rightarrow T_{\mathrm{c}} .
\end{gathered}
$$

Of course, on finite lattices there is no singularity. In the three-dimensional Ising model, the critical exponents were calculated from theoretical models [4] as well as from Monte Carlo simulations [5-7] with the result

$$
\beta \simeq 0.325, \quad \gamma \simeq 1.24, \quad \nu \simeq 0.63, \quad \alpha \approx 0.11 .
$$

The check of the universality hypothesis consists now of a comparison with these numbers.

Our data were taken on $N_{\sigma}^{3} \times 4$ lattices with $N_{\sigma}=8,12,18$ and 26. In general, we used 100000 sweeps per point, very close to $T_{\mathrm{c}}$, this was increased up to 450000 updates per point. The evaluation was carried out with a full group heat-bath vector program. For thermalization, we discarded the first 1000 iterations before data measuring.

In fig. 1 we show the probability density $P(|L|)$ of the modulus of the lattice average of the Polyakov loop at $4 / g^{2}=2.27,2.29,2.30,2.31$ and 2.35 . This corresponds to $T / \Lambda_{\mathrm{L}}=39.21,41.22,42.27,43.34$ and 47.92 ; the critical point is at 42.11, as we shall see. Let us first look at the results for the largest, the $26^{3} \times 4$ lattice. Well above $T_{\mathrm{c}}$ we have a single gaussian peak. Approaching $T_{\mathrm{c}}$ the peak broadens and becomes asymmetric, the region between the position of the maximum, $|L|_{\max }$ and $|L|=0$ is being filled up due to the increasing probability for tunnelling between the two equivalent broken symmetry states. Even very close to $T_{\mathrm{c}}$ we still have a maximum of the distribution for $|L|>0$, well below $T_{\mathrm{c}}$ a gaussian distribution around $|L|=0$ is obtained. On the smaller lattices we find in principal the same behaviour; the main differences are

(i) the heights of the maxima decrease, the widths of the distributions increase with decreasing $N_{\sigma}$ (see the different scales in the plots),

(ii) the smaller the lattice is, the larger is the tunnelling probability above $T_{\mathrm{c}}$, i.e. in the distributions the region below $|L|_{\max }$ is enhanced,

(iii) with decreasing $N_{\sigma}$ the point $|L|_{\max }$ is increasing. Obviously it is very difficult to determine the critical point on too small lattices; even below $T_{\mathrm{c}}$ the distribution may still have a maximum at $|L| \neq 0$. It is important to note, that all distributions show only one peak, in contrast to what is observed at a first order transition.

Since, due to the system flips between the two ordered states on finite lattices the expectation value $\langle L\rangle$ is always zero, we take as "order parameter" the expectation 


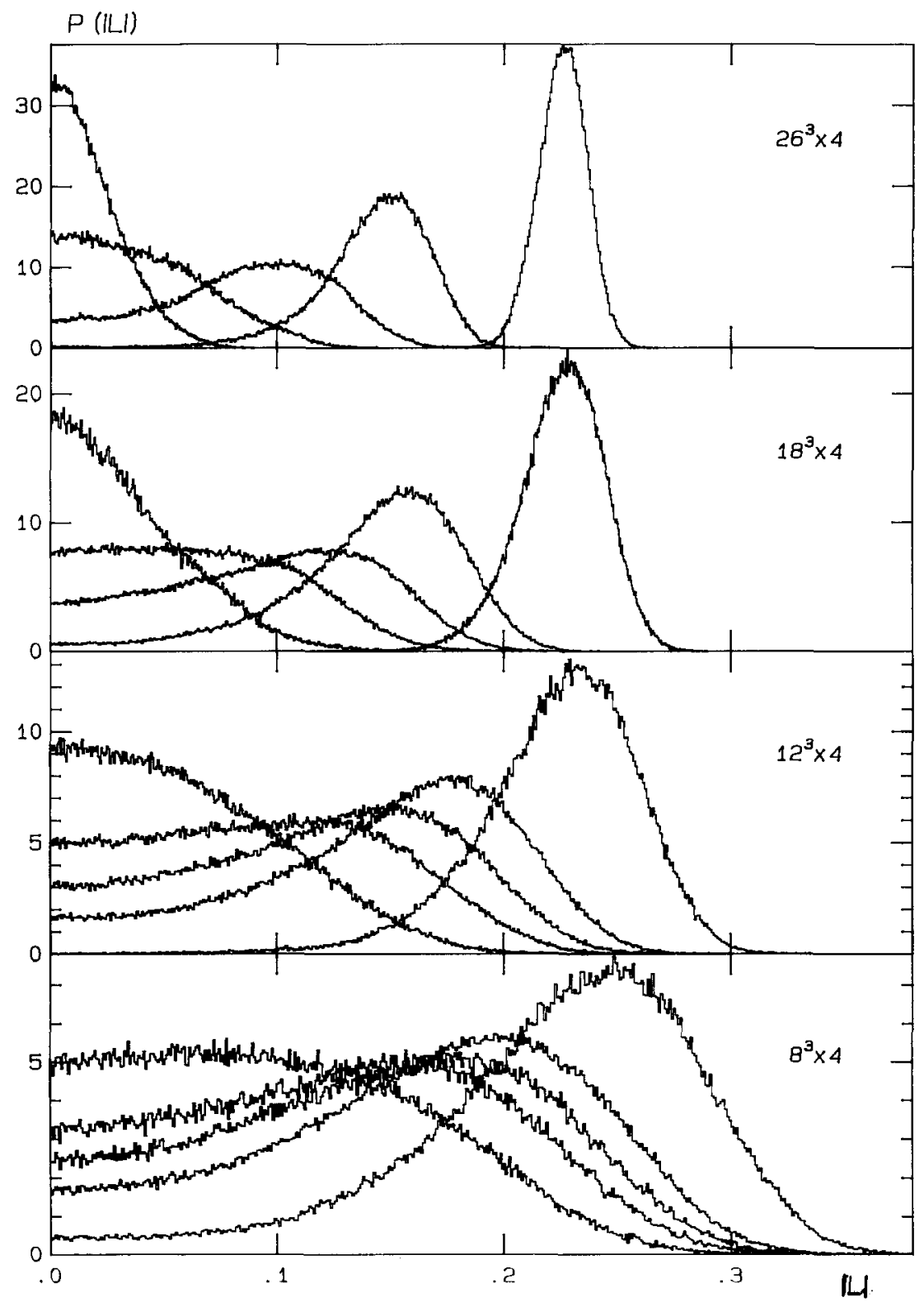

Fig. 1. The probability density $P(|L|)$ for the $8^{3} \times 4,12^{3} \times 4,18^{3} \times 4$ and $26^{3} \times 4$ lattices at $4 / g^{2}=2.27$, $2.29,2.30,2.31$ and 2.35 . The histograms with the maximum at $|L|=0$ correspond to $4 / g^{2}=2.27$, the ones with maximum at $|L|>0.22$ to $4 / \mathrm{g}^{2}=2.35$ 


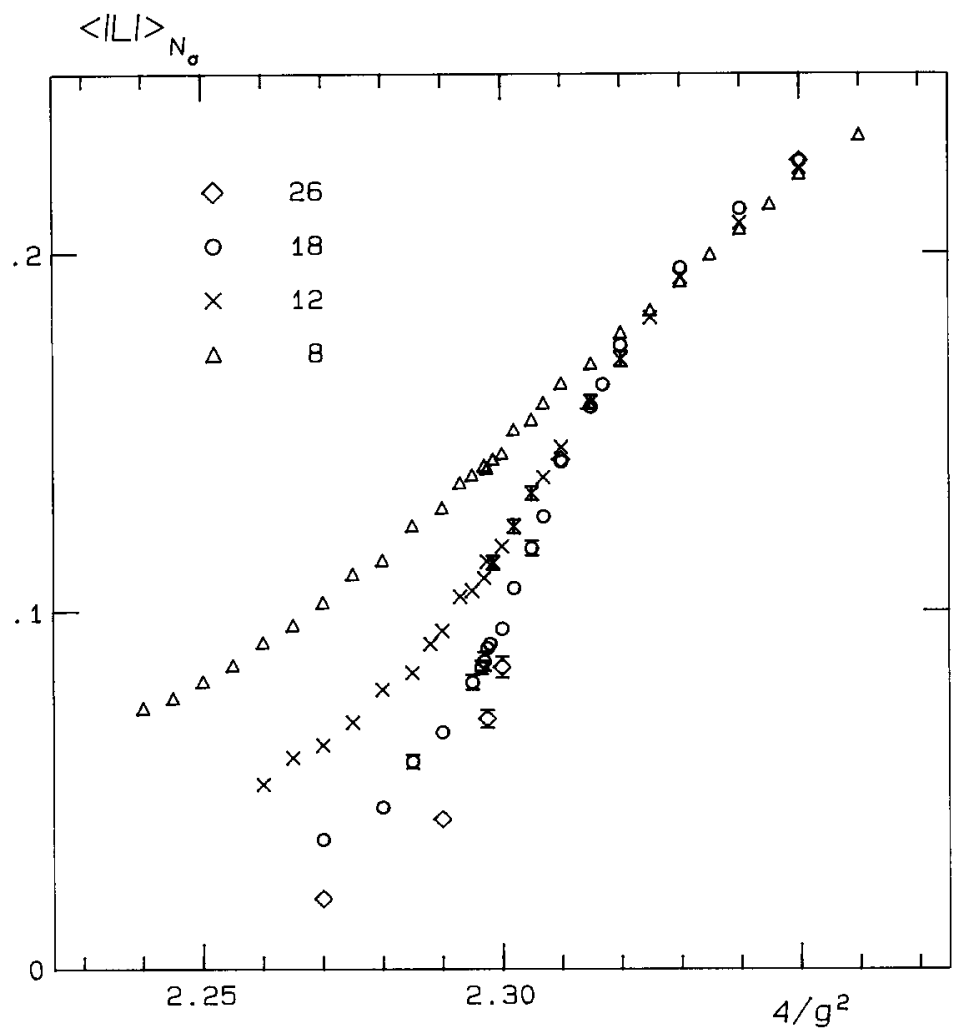

Fig. 2. The expectation value of the modulus of the lattice average for different lattice sizes.

value of the modulus of the lattice average of the Polyakov loop. It is shown in fig. 2. The errors on $\langle|L|\rangle_{N_{\sigma}}$ were calculated both with the blocking method [8] and the method of Daniell et al. [9], which led to the same result. Among the quantities measured, $\langle|L|\rangle_{N_{\sigma}}$ has the least errors. We see from fig. 2 an approach to the limiting form $(6)$ with increasing $N_{\sigma}$. Just above the critical $4 / g^{2}(\approx 2.30)$ each two data sets cross twice, for larger $4 / g^{2}$ the data with bigger $N_{\sigma}$ are smaller, in accord with the weak coupling expansion of $\langle|L|\rangle_{N_{o}}$.

The data for the susceptibility

$$
\chi_{N_{o}}=N_{\mathrm{o}}^{3}\left(\left\langle L^{2}\right\rangle-\langle|L|\rangle^{2}\right)
$$

are plotted in fig. 3. The estimate of the error of the susceptibility is not straightforward, since $\chi$ is composed of two expectation values. We take the average distance of the values of $\chi$ found for four subblocks to the value for the total data set as estimator. As expected for a second order phase transition we find an increasing $\chi_{N_{o}}$ 


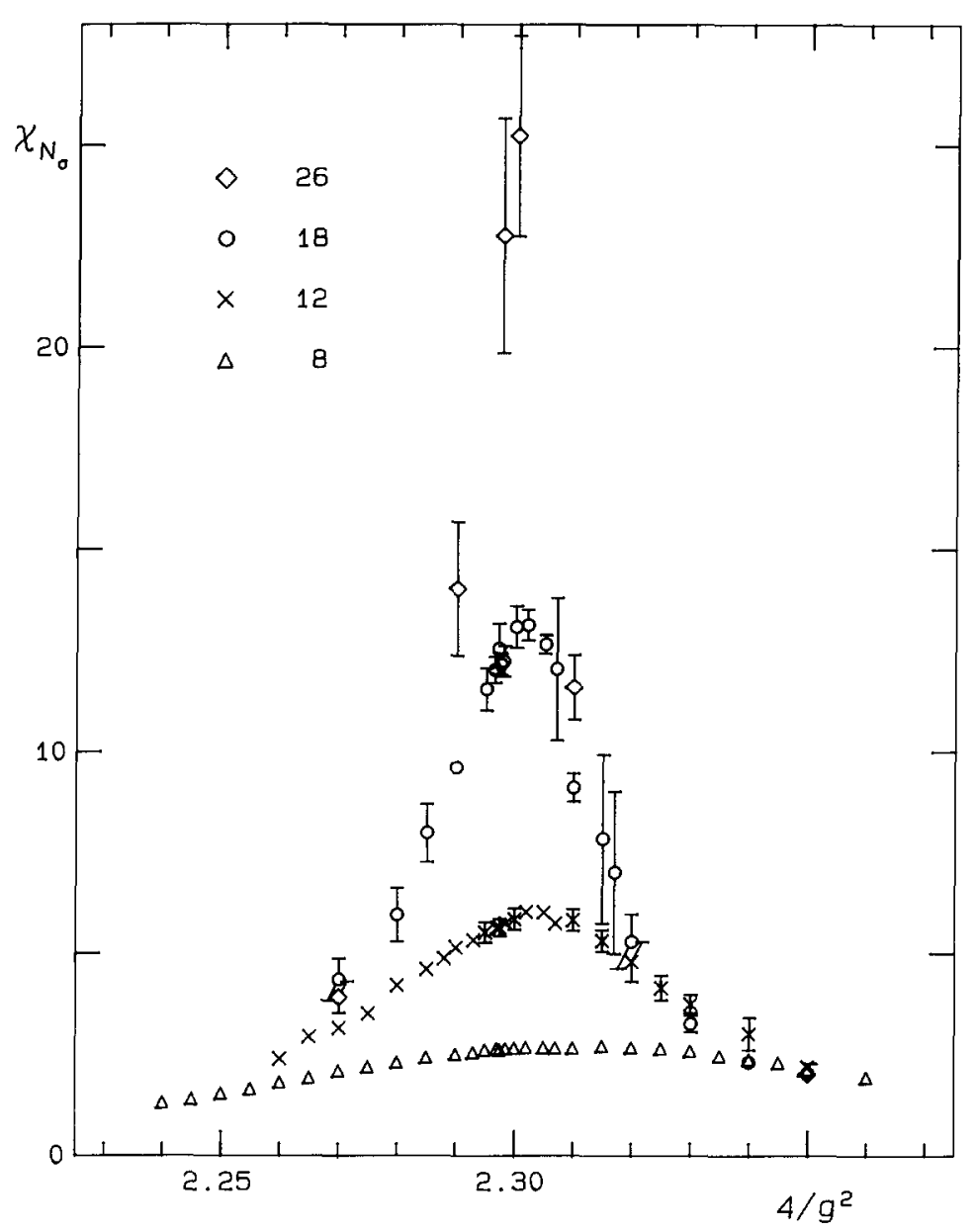

Fig. 3. The susceptibility $\chi$ for different lattice sizes.

with increasing $N_{\mathrm{o}}$ near $T_{\mathrm{c}}$. Somewhat further away from the critical point the different $N_{\sigma}$-curves cross again, $\chi_{N_{\sigma}}$ is then decreasing with increasing $N_{\sigma}$.

The direct calculation of the specific heat $C_{\mathrm{v}}$

$$
C_{\mathrm{v}}=\partial \epsilon / \partial T
$$

requires plaquette correlation measurements or the numerical derivative of the energy density $\epsilon$. Since both methods do not result in very reliable data, we investigate instead quantities connected to the energy density and pressure $P$, namely the entropy density

$$
s / T^{3}=(\epsilon+P) / T^{4}=16\left(N_{\tau}^{4} / g^{2}\right)\left(1+g^{2} c_{\mathrm{G}}^{\prime}\right)\left(\mathrm{P}_{\sigma}-\mathrm{P}_{\tau}\right)
$$




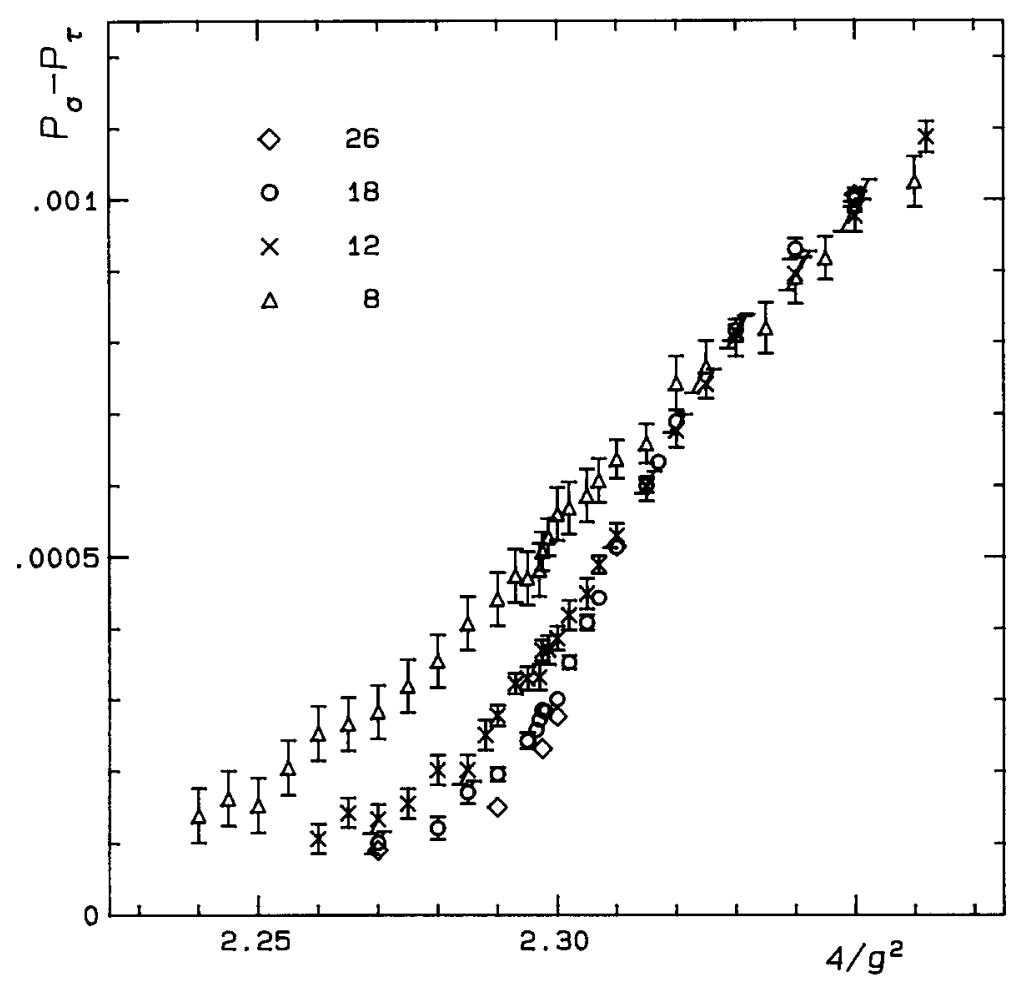

Fig. 4. The difference $P_{\sigma}-P_{\tau}$, which is proportional to the entropy density, for different lattice sizes.

where $c_{\mathrm{G}}^{\prime}=-0.0908$ and $\mathrm{P}_{\sigma}$ and $\mathrm{P}_{\tau}$ are the space-space and space-time plaquettes, and the interaction measure $\Delta$, which is given by

$$
\Delta=(\epsilon-3 P) / T^{4}=24 N_{\tau}^{4} a \frac{\mathrm{d} g^{-2}}{\mathrm{~d} a}\left(\frac{\mathrm{P}_{\sigma}+\mathrm{P}_{\tau}}{2}-\mathrm{P}_{0}\right),
$$

and vanishes for an ideal gas of massless gluons. In the last equation, $\mathrm{P}_{0}$ is the plaquette on a symmetric $\left(N_{\sigma}^{4}\right)$ lattice and was included to take away the $T=0$ contribution. For a discussion of the dynamical implications of the forms of $s / T^{3}$ and $\Delta$ we refer the reader to ref. [10]. Our current interest in these quantities is in their finite size behaviour. For that purpose the $g^{2}$-dependent, but volume-independent factors in eqs. (13) and (14) are not needed. Therefore we have directly plotted the difference of $P_{\sigma}$ and $P_{\tau}$ and the difference of the average of $P_{\sigma}$ and $P_{\tau}$ to $P_{0}$ for the different lattices in figs. 4 and 5 . We observe in the neighbourhood of the critical point again a strong finite size effect similar to the one in $\langle|L|\rangle$.

Due to the relatively large computer time necessary for an update of the $26^{3} \times 4$ lattice, we measured only at eight $4 / g^{2}$-values there. The smaller lattices were 


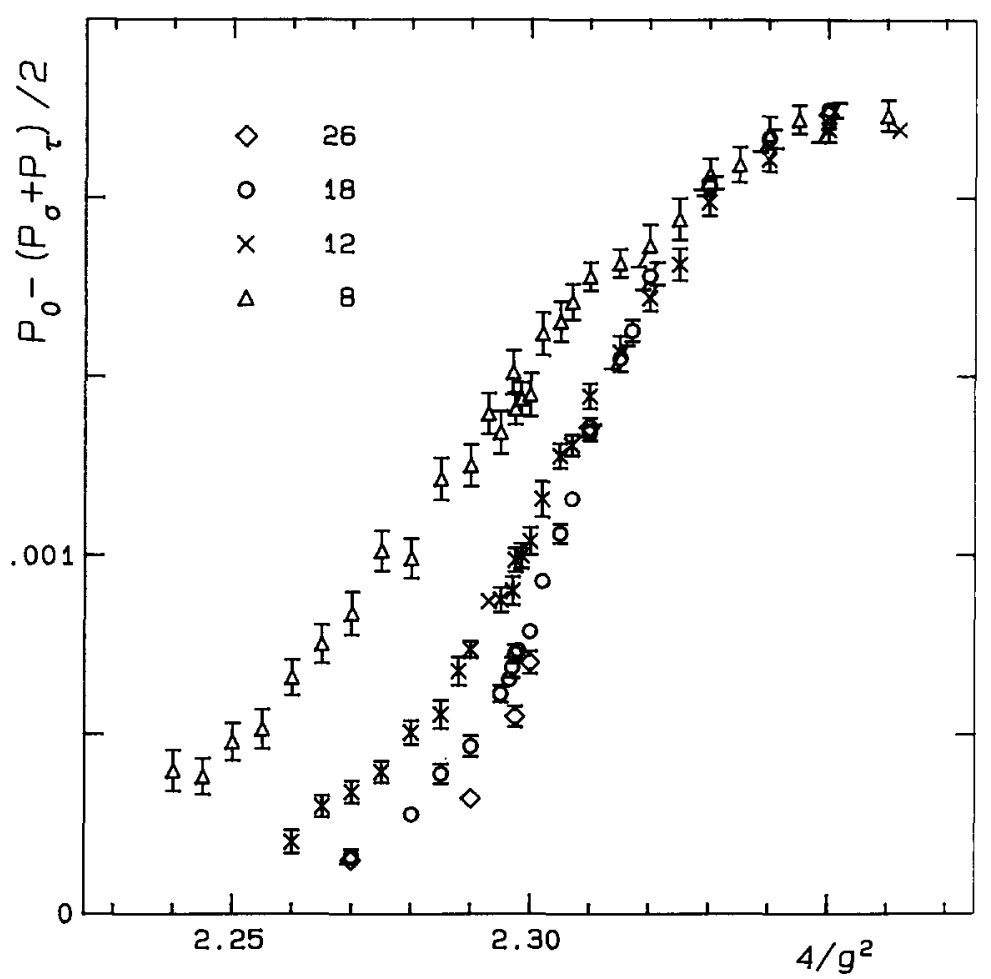

Fig. 5. The difference $\mathrm{P}_{0}-\left(\mathrm{P}_{\sigma}+\mathrm{P}_{\tau}\right) / 2$, which is proportional to the interaction measure $\Delta$, for different lattice sizes.

TABLE 1

Number of iterations in units of 1000

\begin{tabular}{llllll}
\hline $4 / g^{2}$ & $T / \Lambda_{\mathrm{L}}$ & $8^{3} \times 4$ & $12^{3} \times 4$ & $18^{3} \times 4$ & $26^{3} \times 4$ \\
\hline 2.16 & 29.78 & 150 & 151 & 151 & 100 \\
2.21 & 33.74 & 200 & 200 & 200 & 100 \\
2.27 & 39.21 & 200 & 200 & 100 & 150 \\
2.29 & 41.22 & 200 & 400 & 300 & 150 \\
2.2975 & 42.01 & 400 & 401 & 459 & 208 \\
2.30 & 42.27 & 200 & 300 & 450 & 200 \\
2.31 & 43.34 & 400 & 300 & 300 & 150 \\
2.35 & 47.92 & 200 & 150 & 194 & 100 \\
\hline
\end{tabular}


TABLE 2

Polyakov loop values $\langle|L|\rangle$

\begin{tabular}{lccccc}
\hline $4 / g^{2}$ & $T / \Lambda_{\mathrm{L}}$ & $8^{3} \times 4$ & $12^{3} \times 4$ & $18^{3} \times 4$ & $26^{3} \times 4$ \\
\hline 2.16 & 29.78 & $0.0400(03)$ & $0.0218(02)$ & $0.0118(01)$ & $0.0068(01)$ \\
2.21 & 33.74 & $0.0548(05)$ & $0.0299(03)$ & $0.0164(01)$ & $0.0094(01)$ \\
2.27 & 39.21 & $0.1026(12)$ & $0.0625(10)$ & $0.0361(12)$ & $0.0196(05)$ \\
2.29 & 41.22 & $0.1288(14)$ & $0.0945(12)$ & $0.0661(14)$ & $0.0417(17)$ \\
2.2975 & 42.01 & $0.1399(11)$ & $0.1137(13)$ & $0.0897(15)$ & $0.0698(25)$ \\
2.30 & 42.27 & $0.1440(15)$ & $0.1180(16)$ & $0.0951(16)$ & $0.0843(30)$ \\
2.31 & 43.34 & $0.1637(10)$ & $0.1458(16)$ & $0.1417(14)$ & $0.1423(14)$ \\
2.35 & 47.92 & $0.2219(13)$ & $0.2236(09)$ & $0.2254(03)$ & $0.2256(03)$ \\
\hline
\end{tabular}

TABLE 3

The susceptibility $\chi$

\begin{tabular}{llllrl}
\hline $4 / g^{2}$ & $T / \Lambda_{\mathrm{L}}$ & $8^{3} \times 4$ & $12^{3} \times 4$ & $18^{3} \times 4$ & $26^{3} \times 4$ \\
\hline 2.16 & 29.78 & $0.46(01)$ & $0.46(01)$ & $0.46(01)$ & $0.46(02)$ \\
2.21 & 33.74 & $0.83(01)$ & $0.86(01)$ & $0.88(02)$ & $0.89(03)$ \\
2.27 & 39.21 & $2.09(03)$ & $3.15(09)$ & $4.36(52)$ & $3.92(39)$ \\
2.29 & 41.22 & $2.50(05)$ & $5.15(07)$ & $9.61(10)$ & $14.03(165)$ \\
2.2975 & 42.01 & $2.61(02)$ & $5.64(21)$ & $12.16(27)$ & $22.77(290)$ \\
2.30 & 42.27 & $2.65(03)$ & $5.85(26)$ & $13.09(52)$ & $25.23(248)$ \\
2.31 & 43.34 & $2.65(04)$ & $5.82(27)$ & $9.11(35)$ & $11.59(79)$ \\
2.35 & 47.92 & $2.11(16)$ & $2.19(09)$ & $2.03(05)$ & $2.01(13)$ \\
\hline
\end{tabular}

TABle 4

The difference $P_{\sigma}-P_{\tau}$ in units of $10^{-4}$

\begin{tabular}{lrrrrr}
\hline $4 / g^{2}$ & $T / \Lambda_{\mathrm{L}}$ & $8^{3} \times 4$ & $12^{3} \times 4$ & $18^{3} \times 4$ & $26^{3} \times 4$ \\
\hline 2.16 & 29.78 & $0.03(46)$ & $0.18(25)$ & $0.31(14)$ & $0.27(10)$ \\
2.21 & 33.74 & $0.73(38)$ & $0.37(21)$ & $0.37(12)$ & $0.39(09)$ \\
2.27 & 39.21 & $2.83(37)$ & $1.34(20)$ & $1.01(16)$ & $0.90(07)$ \\
2.29 & 41.22 & $4.42(37)$ & $2.77(15)$ & $1.96(10)$ & $1.50(08)$ \\
2.2975 & 42.01 & $5.08(27)$ & $3.70(15)$ & $2.85(07)$ & $2.32(07)$ \\
2.30 & 42.27 & $5.60(37)$ & $3.87(17)$ & $3.01(08)$ & $2.76(06)$ \\
2.31 & 43.34 & $6.37(27)$ & $5.30(17)$ & $5.14(09)$ & $5.15(08)$ \\
2.35 & 47.92 & $9.91(36)$ & $9.77(23)$ & $10.00(11)$ & $10.06(10)$ \\
\hline
\end{tabular}


TABLE 5

The difference $\mathrm{P}_{0}-\left(\mathrm{P}_{\boldsymbol{\sigma}}+\mathrm{P}_{r}\right) / 2$ in units of $10^{-4}$

\begin{tabular}{lrrrrr}
\hline $4 / g^{2}$ & $T / \Lambda_{\mathrm{L}}$ & $8^{3} \times 4$ & $12^{3} \times 4$ & $18^{3} \times 4$ & $26^{3} \times 4$ \\
\hline 2.16 & 29.78 & $0.19(51)$ & $0.14(29)$ & $0.08(15)$ & $0.15(15)$ \\
2.21 & 33.74 & $2.24(47)$ & $0.52(28)$ & $0.57(17)$ & $0.43(15)$ \\
2.27 & 39.21 & $8.39(60)$ & $3.39(31)$ & $1.59(20)$ & $1.49(13)$ \\
2.29 & 41.22 & $12.53(58)$ & $7.37(23)$ & $4.67(29)$ & $3.22(19)$ \\
2.2975 & 42.01 & $14.12(44)$ & $9.87(33)$ & $7.25(25)$ & $5.50(29)$ \\
2.30 & 42.27 & $14.50(60)$ & $10.39(39)$ & $7.88(16)$ & $7.01(31)$ \\
2.31 & 43.34 & $17.81(40)$ & $14.46(45)$ & $13.44(22)$ & $13.59(26)$ \\
2.35 & 47.92 & $22.11(55)$ & $21.91(34)$ & $22.47(18)$ & $22.33(16)$ \\
\hline
\end{tabular}

TABLE 6

The renormalized coupling $g_{\mathrm{r}}$

\begin{tabular}{lccccc}
\hline $4 / g^{2}$ & $T / \Lambda_{\mathrm{L}}$ & $8^{3} \times 4$ & $12^{3} \times 4$ & $18^{3} \times 4$ & $26^{3} \times 4$ \\
\hline 2.16 & 29.78 & $-0.088(25)$ & $-0.029(52)$ & $-0.031(38)$ & $-0.004(64)$ \\
2.21 & 33.74 & $-0.212(31)$ & $-0.105(21)$ & $-0.025(117)$ & $0.044(91)$ \\
2.27 & 39.21 & $-0.916(57)$ & $-0.598(30)$ & $-0.103(51)$ & $-0.036(120)$ \\
2.29 & 41.22 & $-1.226(20)$ & $-1.120(28)$ & $-0.971(33)$ & $-0.647(142)$ \\
2.2975 & 42.01 & $-1.323(27)$ & $-1.351(28)$ & $-1.336(15)$ & $-1.286(135)$ \\
2.30 & 42.27 & $-1.352(16)$ & $-1.387(35)$ & $-1.374(51)$ & $-1.479(69)$ \\
2.31 & 43.34 & $-1.503(26)$ & $-1.606(23)$ & $-1.788(14)$ & $-1.901(08)$ \\
2.35 & 47.92 & $-1.770(16)$ & $-1.916(03)$ & $-1.974(01)$ & $-1.991(01)$ \\
\hline
\end{tabular}

evaluated at $25-40$ points. The number of iterations and the results for the $4 / g^{2}$-values with the complete set of data are presented in tables 1-6.

\section{Finite size scaling theory}

\subsection{DIRECT SCALING ANSATZ}

On a finite lattice the correlation length is limited by the characteristic length scale of the system, in our case $N_{\mathrm{o}}$. That observation leads to the introduction of a scaled variable $[11,12]$

$$
y=N_{\sigma} / \xi,
$$

where $\xi$ is the correlation length of the infinite volume lattice. Near a second order transition $\xi$ is supposed to diverge as described by eq. (8). If instead of the 
temperature we use a normalized variable

$$
x=\frac{T-T_{\mathrm{c}, \infty}}{T_{\mathrm{c}, \infty}}, \quad \text { or } \quad x=\frac{4 / g^{2}-4 / g_{\mathrm{c}, \infty}^{2}}{4 / g_{c, \infty}^{2}}
$$

any observable $O$ with critical behaviour will have the form

$$
O_{\infty} \sim x^{-\rho}, \text { for } x \rightarrow 0
$$

On a finite lattice, i.e. for finite $N_{\sigma}$, and for fixed $T$, or $4 / g^{2}$, close to $T_{c, \infty}$, we make then the ansatz $[11,12]$

$$
O_{N_{o}}(T)=N_{\sigma}^{\omega} \bar{Q}_{o}(y)
$$

Here we have neglected correction-to-scaling terms. The behaviour of the correlation length

$$
\xi \sim x^{-\nu}
$$

implies then

$$
y \sim N_{\sigma} x^{\nu}
$$

and we may change variables in eq. (18) to find

$$
O_{N_{\sigma}}=N_{\sigma}^{\omega} Q_{o}\left(x N_{\sigma}^{1 / \nu}\right)
$$

The scaling function $Q_{O}$ must then behave as

$$
Q_{O} \sim\left(x N_{\sigma}^{1 / \nu}\right)^{-\rho}
$$

for fixed small $x$, if the form (17) is to be recovered for $N_{\sigma} \rightarrow \infty$, the exponent $\omega$ must be

$$
\omega=\rho / \nu
$$

The ansatz for $O_{N_{\sigma}}$ may be tested in the following way: one plots $O_{N_{o}} N_{\sigma}^{-\rho / \nu}$ as a function of $x N_{\sigma}^{1 / \nu}$. For large $T$ near $T_{\mathrm{c}, \infty}$, and $N_{\sigma}$ large one should get a unique function independent of $N_{\sigma}$. Of course, such scaling plot tests may be carried out independently for each observable, no hyperscaling relations between different critical exponents have to be known as input. On the other hand, there must be relations between different critical exponents, because the critical behaviour of all observables is determined by the same partition function. 


\subsection{SCALING ANSATZ FOR THE SINGULAR PART OF THE FREE ENERGY}

The basic idea of this approach [11] is to consider the singular part of the free energy density

$$
f_{\mathrm{s}}=\frac{F_{\mathrm{s}}}{V T}=-\frac{1}{V} \ln Z_{\mathrm{s}}
$$

and to assume a scaling ansatz for this quantity

$$
f_{\mathrm{s}}\left(x, h, N_{\sigma}\right)=N_{\sigma}^{-d} Q\left(g_{\mathrm{T}} N_{\sigma}^{1 / \nu}, g_{h} N_{\sigma}^{(\beta+\gamma) / \nu}, g_{i} N_{\sigma}^{y_{i}}\right),
$$

where $d$ is the spatial dimension. The scaling function $Q$ depends on the thermal scaling field

$$
g_{\mathrm{T}}=c_{\mathrm{T}} x+\mathrm{O}\left(x^{2}\right)
$$

and the magnetic scaling field

$$
g_{h}=c_{h} h+\mathrm{O}(x h)
$$

here $c_{\mathrm{T}}$ and $c_{h}$ are constants and $h$ denotes the coupling of the symmetry breaking term $h \sum_{x} L(x)$ in the action. The additional dependence on irrelevant scaling fields $g_{i}$ with $y_{i}<0$ determines the correction-to-scaling terms.

The order parameter and the susceptibility are now obtained as derivatives of $f_{\mathrm{s}}$

$$
\begin{gathered}
\langle L\rangle=-\left.\frac{\partial f_{\mathrm{s}}}{\partial h}\right|_{h=0}=N_{\sigma}^{-\beta / \nu} Q_{\mathrm{L}}\left(g_{\mathrm{T}} N_{\sigma}^{1 / \nu}, g_{i} N_{\sigma}^{y_{i}}\right), \\
\chi=\left.\frac{\partial^{2} f_{\mathrm{s}}}{\partial h^{2}}\right|_{h=0}=N_{\sigma}^{\gamma / \nu} Q_{\chi}\left(g_{\mathrm{T}} N_{\sigma}^{1 / \nu}, g_{i} N_{\sigma}^{y_{i}}\right) .
\end{gathered}
$$

In the last two equations we have used the hyperscaling relation

$$
\gamma / \nu+2 \beta / \nu=d
$$

Taking the fourth derivative of $f_{\mathrm{s}}$ at $h=0$, it is then easy to see that the quantity

$$
g_{r}=\left.\frac{\partial^{4} f_{\mathrm{s}}}{\partial h^{4}}\right|_{h=0} \mid \chi^{2} N_{o}^{d}
$$

is directly a scaling function

$$
g_{\mathrm{r}}=Q_{g_{\mathrm{r}}}\left(g_{\mathrm{T}} N_{\sigma}^{1 / \nu}, g_{i} N_{\sigma}^{y_{i}}\right) .
$$


On a finite lattice $g_{\mathrm{r}}$ has the form

$$
g_{\mathrm{r}}=\left\langle L^{4}\right\rangle /\left\langle L^{2}\right\rangle^{2}-3
$$

i.e. it is the normalized fourth cumulant of the Polyakov loop. It corresponds, up to a constant, to the renormalized coupling defined for infinite systems $[6,13,14]$.

Consider now the energy density

$$
\epsilon=-\left.\frac{1}{V} \frac{\partial \ln Z}{\partial(1 / T)}\right|_{V=\text { const }, h=0} .
$$

The contribution from $f_{\mathrm{s}}$ to $\epsilon$ is

$$
\left.\epsilon_{\mathrm{s}} \sim \frac{\partial f_{\mathrm{s}}}{\partial x}\right|_{h=0}, \quad \text { or } \quad \epsilon_{\mathrm{s}}=N_{\sigma}^{-d+1 / \nu} Q_{\epsilon}\left(g_{\mathrm{T}} N_{\sigma}^{1 / \nu}, g_{i} N_{\sigma}^{y_{i}}\right)
$$

Correspondingly one obtains for the specific heat

$$
\left.C_{\mathrm{v}, \mathrm{s}} \sim \frac{\partial^{2} f_{\mathrm{s}}}{\partial x^{2}}\right|_{h=0}, \quad \text { or } \quad C_{\mathrm{v}, \mathrm{s}}=N_{\sigma}^{-d+2 /{ }^{v}} Q_{C_{\mathrm{v}}}\left(g_{\mathrm{T}} N_{\sigma}^{1 / \nu}, g_{i} N_{\sigma}^{y_{i}}\right) \text {. }
$$

With the second hyperscaling relation

$$
\alpha=2-d \nu,
$$

one recovers the expected $N_{\sigma}$-dependence. However, due to the smallness of the exponent $\alpha$, the analytic parts in $C_{\mathrm{V}}$ and $\epsilon$ are dominating. For the total energy density one anticipates therefore the form

$$
\epsilon=\epsilon_{\text {regular }}+N_{\sigma}^{(\alpha-1) / \nu} Q_{\epsilon}\left(g_{\mathrm{T}} N_{\sigma}^{1 / \nu}\right),
$$

where we neglected the irrelevant scaling fields. The corresponding consideration for the pressure

$$
P=\left.T \frac{\partial \ln Z}{\partial V}\right|_{T=\text { const }, h=0}=\left.T \frac{\ln Z}{V}\right|_{h=0}
$$

leads to

$$
P_{\mathrm{s}}=N_{\sigma}^{-d} Q_{\mathrm{P}}\left(g_{\mathrm{T}} N_{\mathrm{\sigma}}^{1 / \nu}, g_{i} N_{\sigma}^{y_{i}}\right)
$$

In comparison with the energy density the pressure is therefore much less size dependent (for $d=3,-d+1 / \nu \approx-1.4$ ) and this is indeed confirmed by the data [10]. 


\section{Applications}

\subsection{THE DETERMINATION OF THE CRITICAL POINT}

If the quantity $g_{\mathrm{r}}$ is directly a scaling function, it must, at $x=0$, the critical point, be independent of $N_{\sigma}$, apart from corrections to scaling [6]. Expanding $Q_{g_{\mathrm{r}}}$ around $x=0$ results in

$$
g_{\mathrm{r}}\left(x, N_{\sigma}\right)=a_{0}+\left(a_{1}+a_{2} N_{\sigma}^{y_{1}}\right) x N_{\sigma}^{1 / \nu}+a_{3} N_{\sigma}^{y_{1}}+\cdots,
$$

where the $a_{i}$ are constants and one irrelevant scaling field with exponent $y_{1}<0$ has been taken into account. At $x=0$ we get

$$
g_{\mathrm{r}}\left(0, N_{\sigma}\right)=a_{0}+a_{3} N_{\sigma}^{y_{1}} .
$$

For large $N_{\sigma}$ the intersection point for two $N_{\sigma}$-curves is the critical point; no information about the critical exponent $\nu$ is required!

In fig. 6 we have plotted our data for $g_{r}$. They are confined to the range $-2 \leqslant g_{\mathrm{r}} \leqslant 0$, the interval ends are reached in the weak and strong coupling limits.

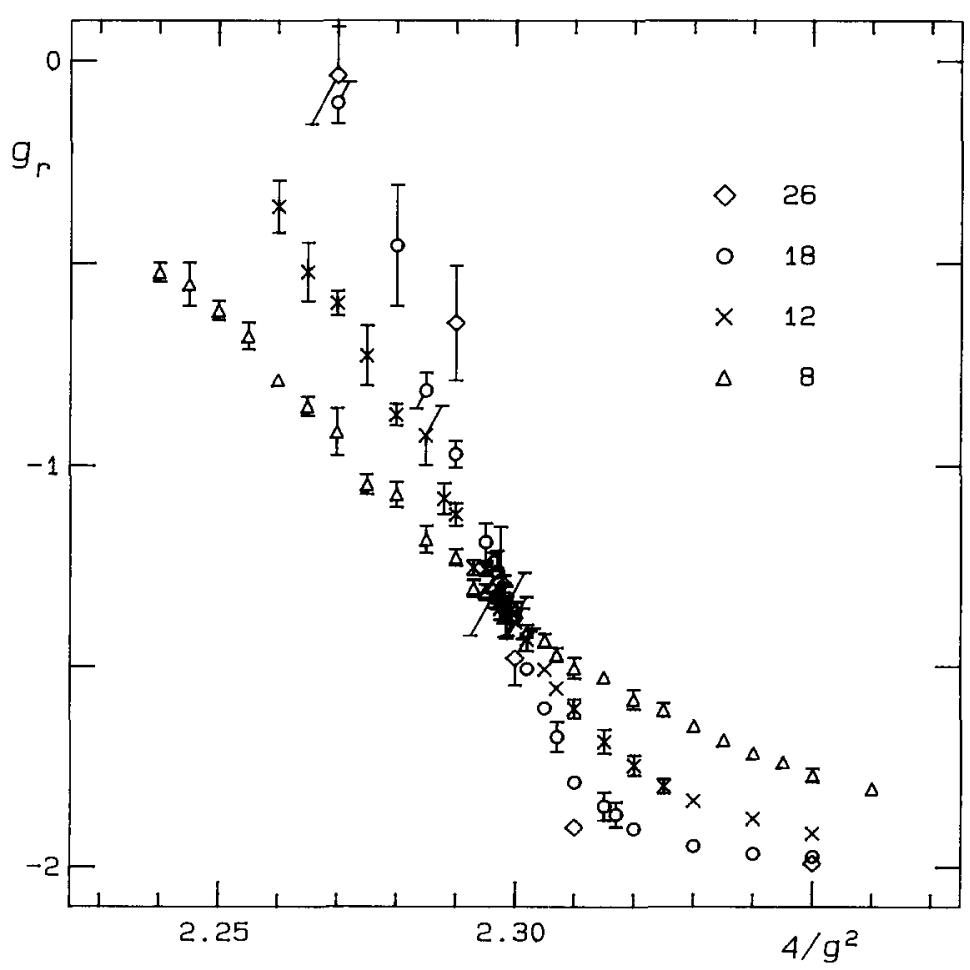

Fig. 6. The normalized fourth cumulant of the Polyakov loop, $g_{\mathrm{r}}$, for different lattice sizes. 


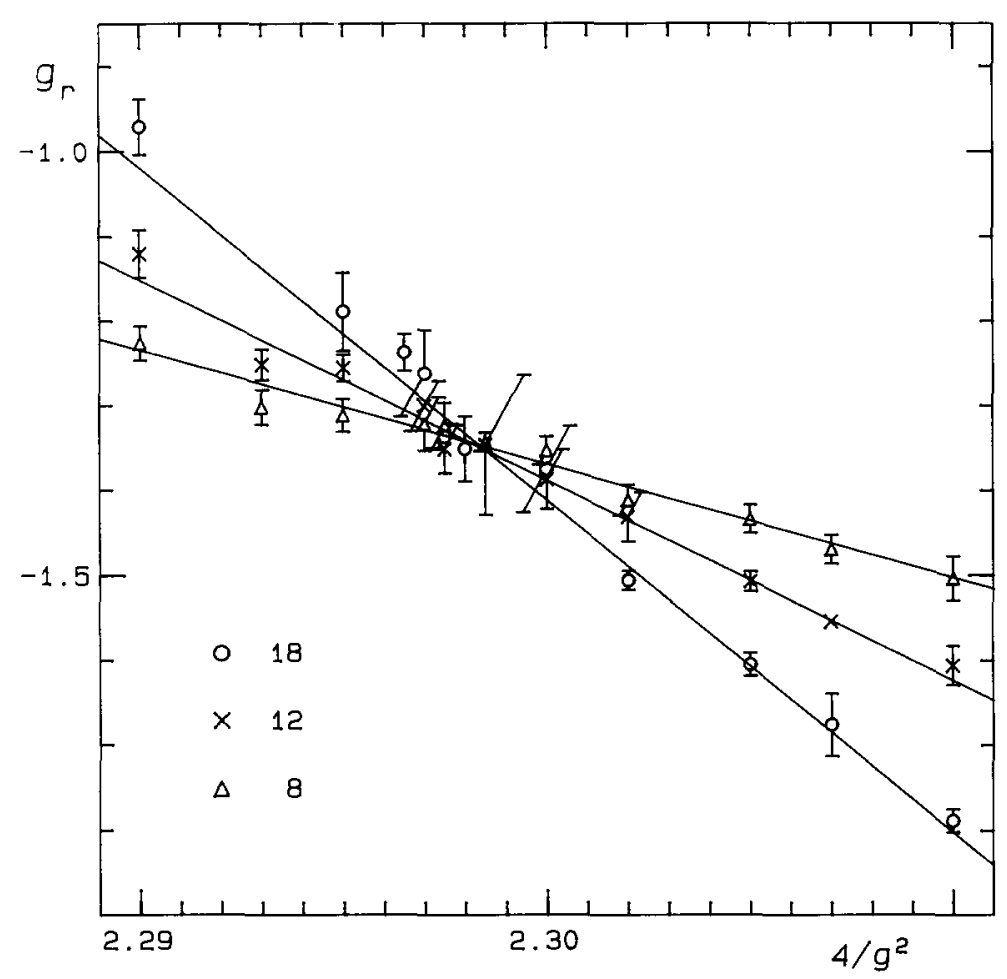

Fig. 7. Enlarged section of fig. 6 , together with straight line fits to the data.

The data for $g_{\mathrm{r}}$ have the largest statistical errors. The estimate of the errors was performed in the same manner as in the case of the susceptibility. We obtain essentially one intersection point for the different $N_{\sigma}$-data, the slope of $g_{\mathrm{r}}$ increases with increasing $N_{\sigma}$. To determine the critical point, we have made linear fits to the data in the region $2.29 \leqslant 4 / g^{2} \leqslant 2.31$, they are shown in fig. 7 . The data for $N_{\sigma}=26$ did not allow for such a fit due to relatively low statistics and too few points. We find as intersection points between each two straight lines

$$
\begin{aligned}
& 4 / g^{2}=2.29812, \text { for } N_{\mathrm{o}}=8-12 \text {, } \\
& 2.29836 \text {, for 8-18, } \\
& 2.29851 \text {, for } 12-18 \text {; }
\end{aligned}
$$

i.e. only a negligible $N_{\sigma}$-dependence. The best value is that of the largest $N_{\sigma}$-values

$$
4 / g_{c, \infty}^{2}=2.2985 \pm 0.0006
$$

its error was estimated with a confidence level of $95 \%$. 


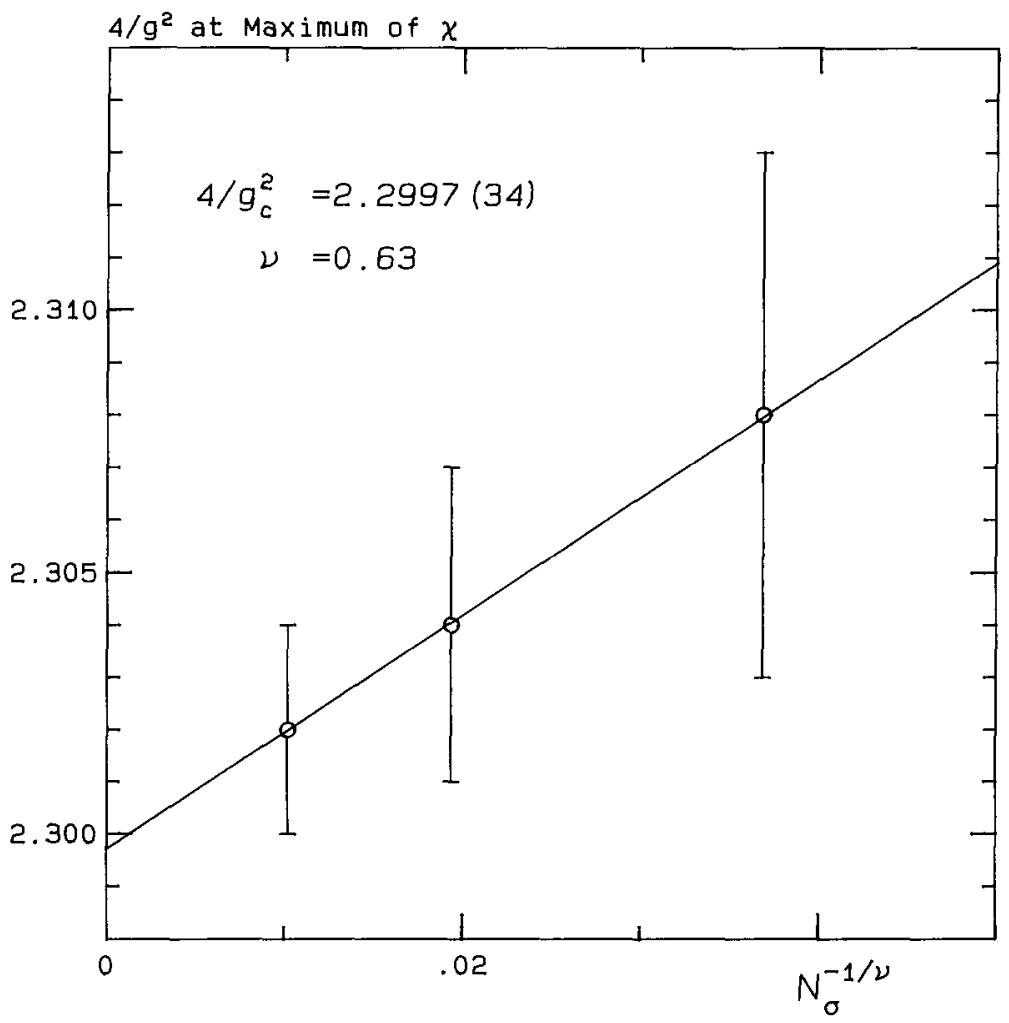

Fig. 8. The $4 / g^{2}$-value of the maximum of the susceptibility $\chi$ versus $N_{\sigma}^{-1 / v}$, with $\nu=0.63$. The straight line is a fit to the data.

A different way for the determination of the infinite volume critical point is the following. The position of the maximum of $\chi_{N_{\sigma}}$ defines a pseudo-critical coupling $4 / g_{\mathrm{c}, N_{\sigma}}^{2}$. The maximum of the scaling function $Q_{\chi}=\left(x N_{\sigma}^{1 / \nu}\right)$ is at a fixed argument

$$
\tilde{x}=x_{c, N_{\sigma}} N_{\sigma}^{1 / \nu} \text {. }
$$

Inserting the definition (16) of $x_{c, N_{o}}$ yields

$$
4 / g_{\mathrm{c}, N_{\mathrm{o}}}^{2}=4 / g_{\mathrm{c}, \infty}^{2}+\tilde{x} \cdot 4 / g_{\mathrm{c}, \infty}^{2} N_{\sigma}^{-1 / \nu}
$$

A plot of the $4 / g_{c, N_{\sigma}}^{2}$ versus $N_{\sigma}^{-1 / \nu}$ may then be extrapolated to $N_{\sigma}^{-1 / \nu}=0$ with a straight line fit. This is shown in fig. 8, the resulting critical point is, with $\nu=0.63$ as 


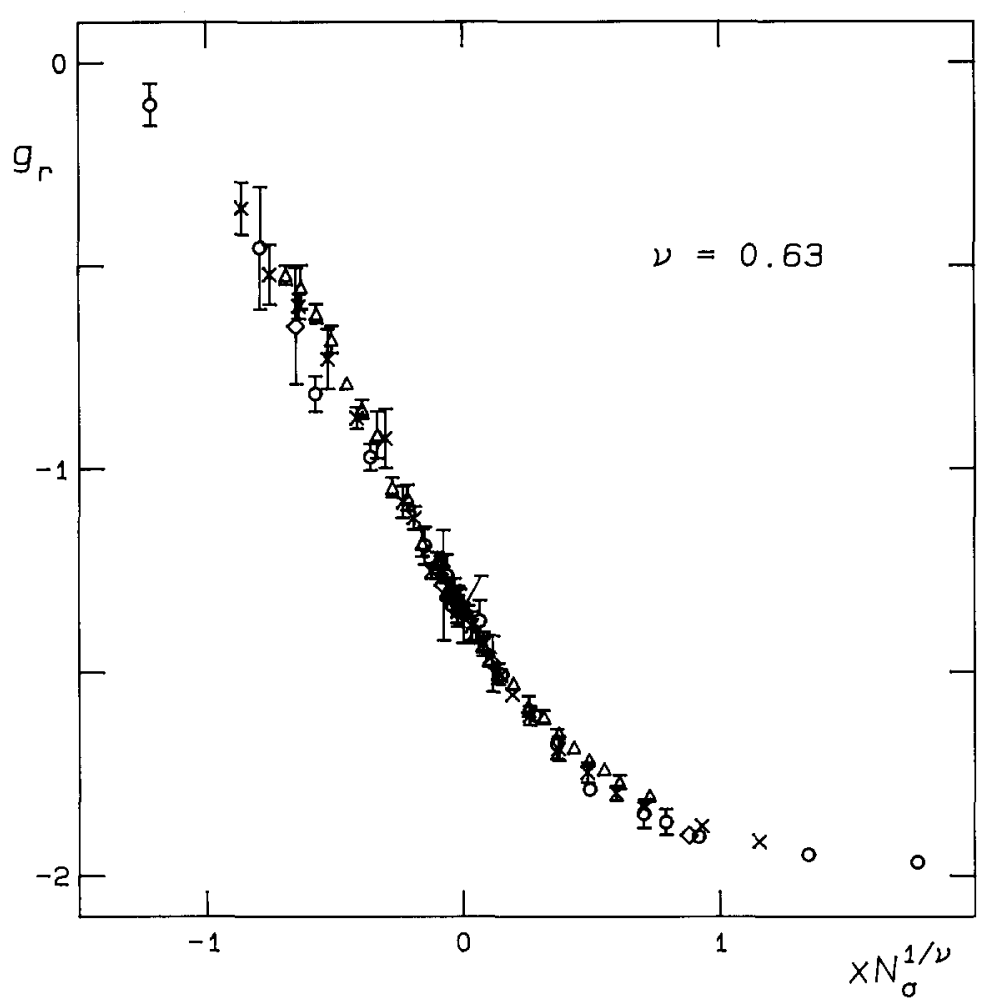

Fig. 9. Scaling plot for $g_{r}$. The critical point was taken from eq. (45), the critical exponent from the three-dimensional Ising model.

input,

$$
4 / g_{c, \infty}^{2}=2.2997 \pm 0.0034
$$

in accord with our previous result (45).

\subsection{THE CHECK OF THE UNIVERSALITY HYPOTHESIS}

To test the predictions for the critical exponents from the universality hypothesis we have plotted in figs. 9-11 the quantities $g_{r},\langle|L|\rangle N_{\sigma}^{\beta / \nu}$ and $\chi N_{\sigma}^{-\gamma / \nu}$ as functions of $x N_{\sigma}^{1 / \nu}$, using the values of the three-dimensional Ising model for $\beta, \gamma$ and $\nu$, as given in eq. (10), and the critical coupling from eq. (45). Obviously the expectations are confirmed in all respects. In fig. 11 the values for $x>0$ are scattering more than for $x<0$. This is due to the tunnel effect above $T_{c}$ and could be improved by an even larger statistics. 


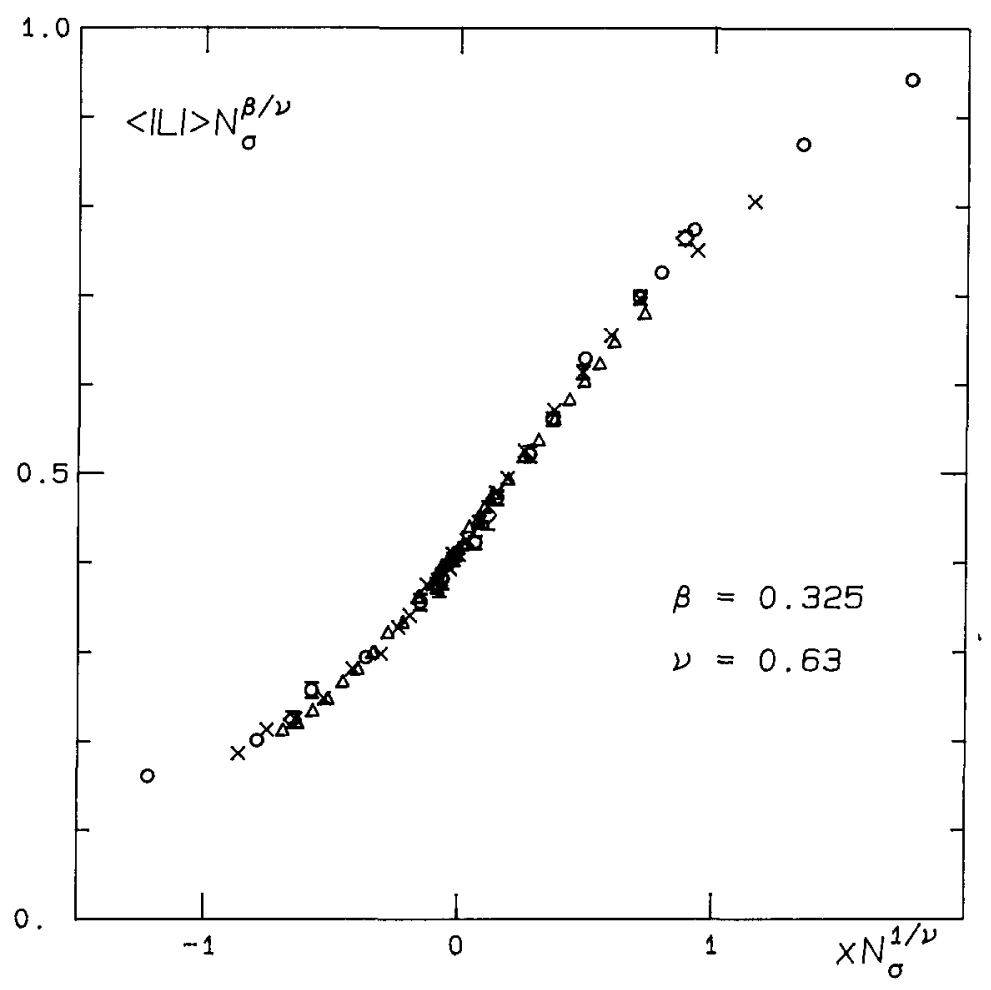

Fig. 10. Scaling plot for $\langle|L|\rangle$, the input is like in fig. 9.

The test of the exponent $\alpha$ is not so straightforward, as is evident from the discussion in subsect. 3.2, because of the dominating analytic parts. In fig. 12 we have plotted $\mathrm{P}_{\sigma}-\mathrm{P}_{\tau} \sim(\epsilon+P) / \mathrm{T}^{4}$ and $\mathrm{P}_{0}-\left(\mathrm{P}_{\sigma}+\mathrm{P}_{\tau}\right) / 2 \sim(\epsilon-3 P) / T^{4}$ at fixed $4 / g^{2}$-values, i.e. fixed $x$, as a function of $N_{\sigma}^{(\alpha-1) / \nu}$, with $\alpha$ and $\nu$ from the Ising model. At the critical point, $x=0$, we expect a straight line. For the range 2.29-2.30 of $4 / g^{2}$ this is in fact the case. Also, in both quantities we find the same functional dependence on $N_{\sigma}^{(\alpha-1) / \nu}$ for the same $4 / g^{2}$-values.

\subsection{SCALING FITS FOR THE CRITICAL EXPONENTS}

The usual methods to determine critical exponents in a finite size scaling analysis require results at the critical point from a larger number of lattices than we have here. On the other hand, we may not only use data at $x=0$, but also in a narrow interval around it, where scaling presumably still works and where we have many data points. The idea is then, to try to find the scaling function $Q\left(x N_{\sigma}^{1 / v}\right)$ with the best scaling behaviour, i.e. with the closest superposition of different $N_{\mathrm{o}}$-data, as a 


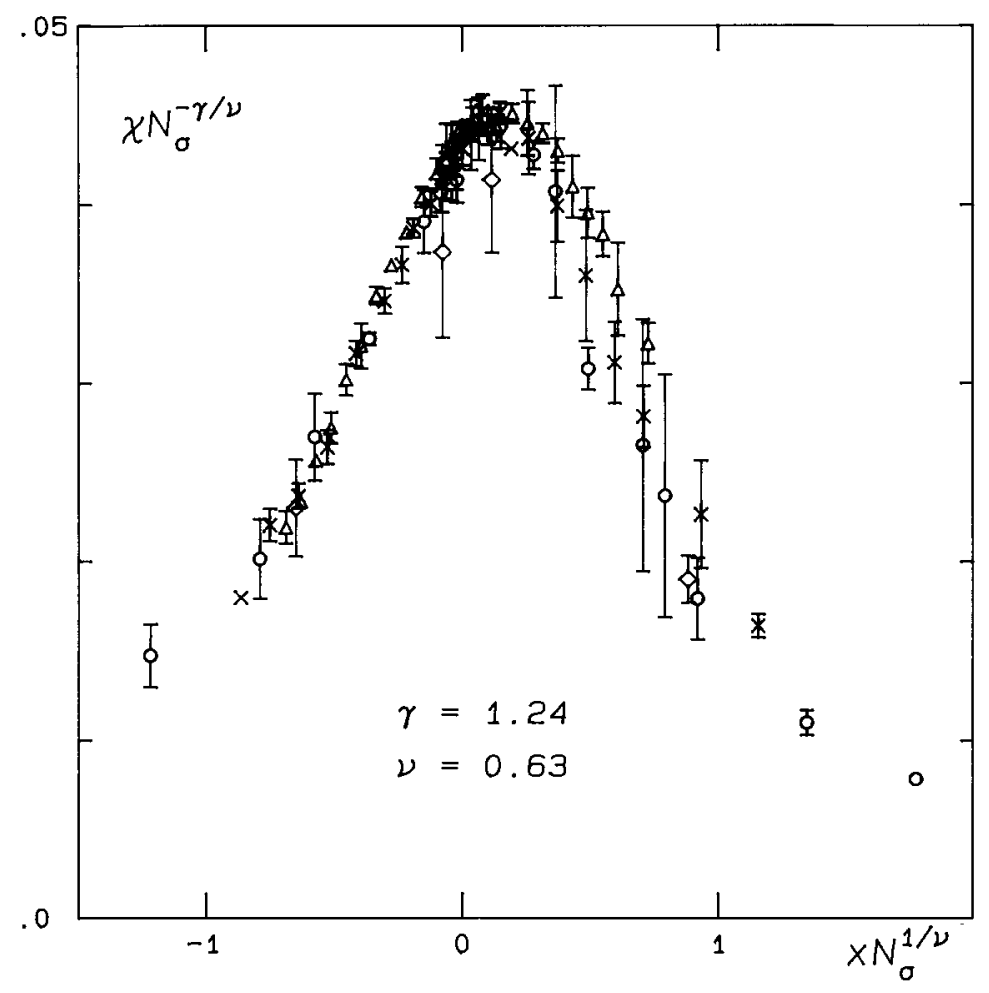

Fig. 11. Scaling plot for $\chi$, the input is like in fig. 9.

function of the exponents and $4 / g_{\mathrm{c}, \infty}^{2}$. Of course, we do not know the functional form of $Q\left(x N_{\sigma}^{1 / \nu}\right)$, but from our experience with the scaling plots in figs. 9-11 we assume that close to $x=0$ the functions $Q_{\mathrm{L}}$ and $Q_{g_{\mathrm{r}}}$ are well approximated by straight lines, the function $Q_{\chi}$ by a parabola.

The data for $\langle|L|\rangle$ have the smallest errors. For straight line $\chi^{2}$-fits with the 20, 25 and 30 closest points to $x=0$ we find

(i) $4 / g_{c, \infty}^{2}=2.2985$, the same value as from the $g_{\mathrm{r}}$-method,

(ii) as a function of $\beta$ and $\nu$ no conclusive result $-\chi^{2}$ is a very flat function; however, varying $\beta / \nu$ at fixed $\nu$ the $\chi^{2}$-minimum is always at the same $\beta / \nu$ :

$$
\beta / \nu=0.545 \pm \begin{cases}0.005 & \text { minimum, } \\ 0.030 & 95 \% \text { confidence level }\end{cases}
$$

and $\quad 0.62 \leqslant \nu \leqslant 0.68$ 


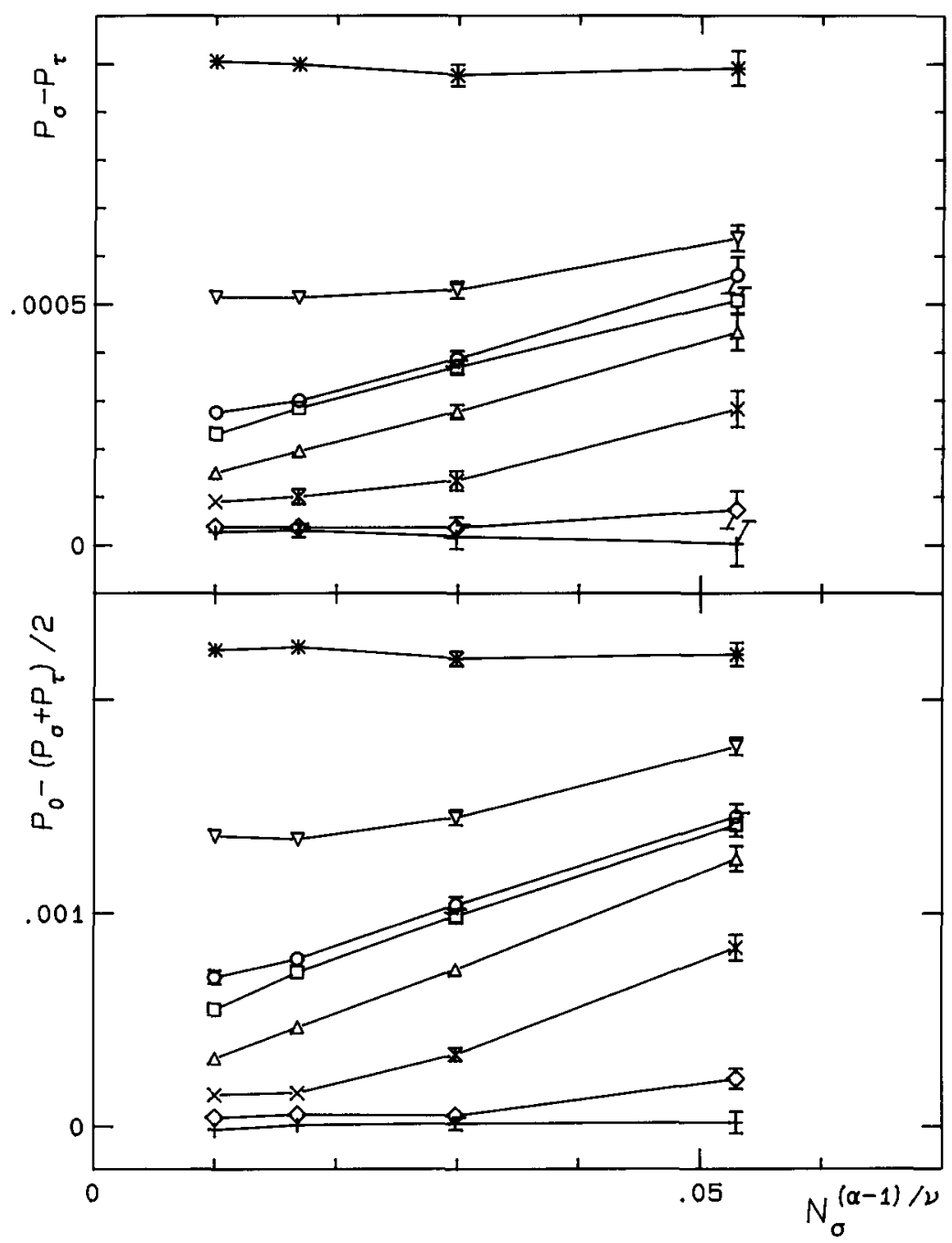

Fig. 12. Test of the exponent $\alpha=0.11$ from the three-dimensional Ising model. $P_{\sigma}-P_{T}$ and $P_{0}-$ $\left(\mathrm{P}_{\sigma}+\mathrm{P}_{\tau}\right) / 2$ are plotted at fixed $4 / \mathrm{g}^{2}=2.16(+), 2.21(\diamond), 2.27(\times), 2.29(\Delta), 2.2975(\square), 2.30(\mathrm{O})$

$2.31(\nabla)$ and $2.35(*)$ versus $N_{a}^{(\alpha-1) / v}$. The points were connected by straight lines to guide the eye. 


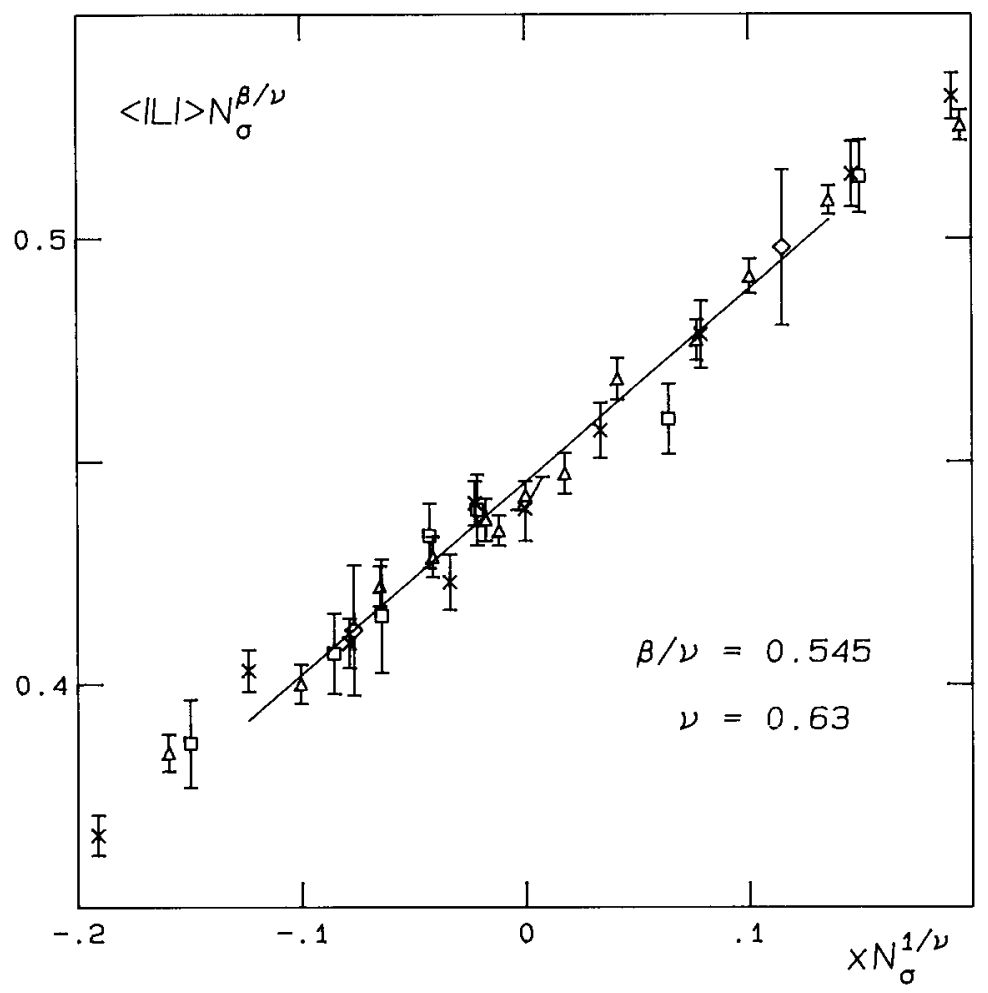

Fig. 13. Scaling fit for $\langle|L|\rangle$. The straight line is the best fit at $\nu=0.63$ to the 25 points closest to $x=0$.

As an example we show in fig. 13 such a fit.

The parabola $\chi^{2}$-fits to the $\chi$-data give correspondingly a well-determined exponent ratio $\gamma / \nu$ :

$$
\gamma / \nu=1.93 \pm \begin{cases}0.01 & \text { minimum, } \\ 0.03 & 95 \% \text { confidence level }\end{cases}
$$

whereas $\nu$ again remains relatively undetermined in the range (50). In fig. 14 such a fit is shown.

The quantity $g_{\mathrm{r}}$ depends only on the exponent $\nu$ and scaling fits of the above kind are possible in the whole range (50). A better determination of $\nu$ requires most probably direct data for the correlation length $\xi$ or rather for Polyakov loop correlations. We have already gathered these data but still not evaluated them.

With the exponent ratios we are now able to test the hyperscaling relation (30):

$$
\gamma / \nu+2 \beta / \nu=3.02 \pm 0.02
$$




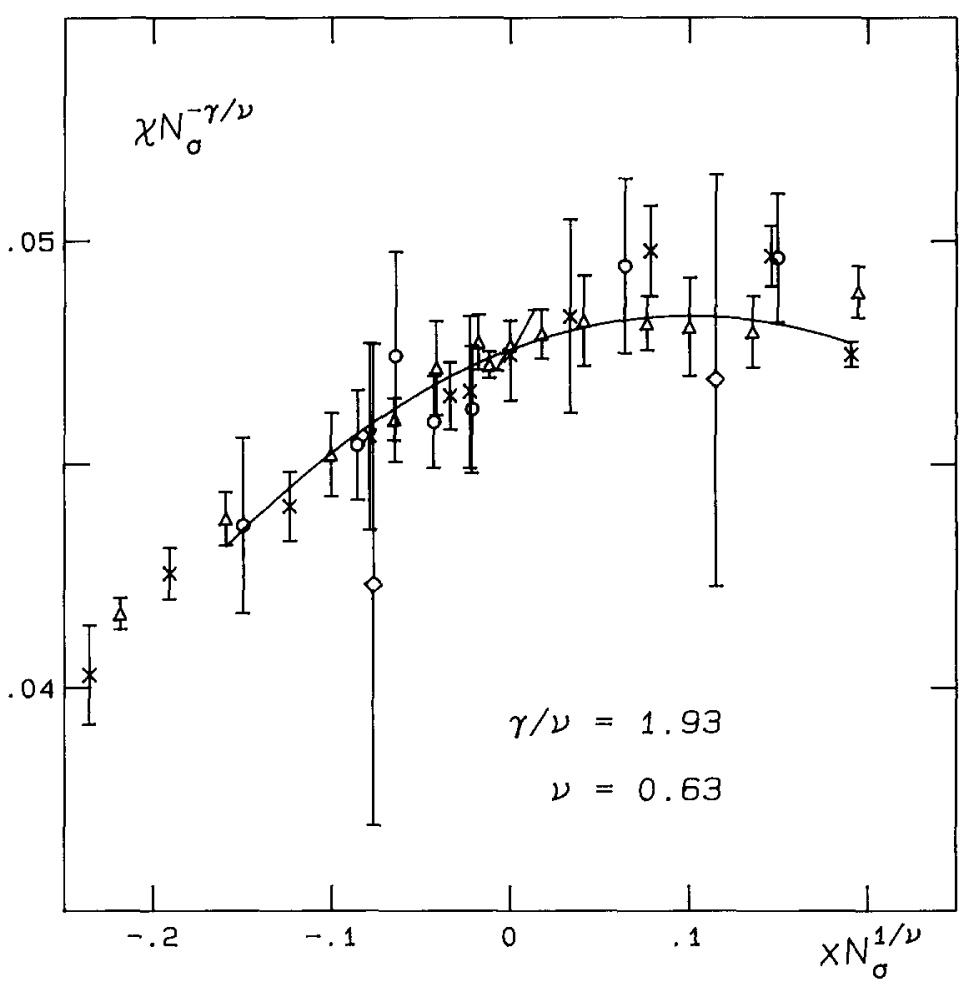

Fig. 14. Scaling fit for $\chi$. The curve is the best fit parabola at $\nu=0.63$ to the 30 points closest to $x=0$.

where the error is from the minimum condition. The result is in excellent agreement with eq. (30).

\section{Summary}

We have investigated the thermodynamical quantities with critical behaviour in SU(2) gauge theory near the deconfinement transition. The volume dependence of these quantities is as expected from finite size scaling theory for a second order phase transition. Moreover, through scaling plots, we have checked the validity of the universality hypothesis of Svetitsky and Yaffe [3], which postulates the coincidence of the critical exponents with those of the three-dimensional Ising model. Our finite size scaling analysis enabled us to determine the infinite volume critical coupling on $N_{\tau}=4$ lattices with very high precision. By direct scaling fits we obtain the ratios $\beta / \nu$ and $\gamma / \nu$, the critical exponent $\nu$ of the correlation length is, however, not very well fixed by these fits

$$
\beta / \nu=0.545 \pm 0.030, \quad \gamma / \nu=1.93 \pm 0.03, \quad \nu=0.65 \pm 0.04 .
$$


The corresponding values of the three-dimensional Ising model are

$$
\beta / \nu=0.516 \pm 0.005, \quad \gamma / \nu=1.965 \pm 0.005, \quad \nu=0.63 \pm 0.003 .
$$

Though our best fit values deviate from those of the Ising model, they fulfill the corresponding hyperscaling relation very well. Inside the error bars we find consistency with the Ising model.

The experience we have gained in our study should be helpful in the assessment of the order and the behaviour of phase transitions of other lattice theories.

It is a pleasure to thank F. Karsch, K. Redlich and H. Satz for many helpful discussions. We are indebted to the HLRZ, Jülich, where most of the calculations were carried out, and to the Bochum University computer centre for providing the necessary computer time.

\section{References}

[1] A. Ukawa, CERN preprint CERN-TH 5245/88

[2] P. Bacilierie et al., Phys. Rev. Lett. 61 (1988) 1545; Rome preprint ROM2F-88-022

[3] B. Svetitsky and G. Yaffe, Nucl. Phys. B210 [FS6] (1982) 423

[4] J.C. le Guillou and J. Zinn-Justin, Phys. Rev. Lett. 39 (1977) 95; Phys. Rev. B21 (1980) 3976; J. Phys. Lett. 46 (1985) L137

[5] K. Binder, Z. Phys. B43 (1981) 119

[6] M.N. Barber, R.B. Pearson, D. Toussaint and J.L. Richardson, Phys. Rev. B32 (1985) 1720

[7] G. Bhanot, D. Duke and R. Salvador, Phys. Rev. B33 (1986) 7841

[8] W.W. Wood, Physics of simple liquids (Wiley, New York, 1968) ch. 5;

R. Friedberg and J.E. Cameron, J. Chem. Phys. 52 (1970) 6049;

D.P. Landau, Phys. Rev. B13 (1976) 2997

[9] G. Daniell, A. Hey and J. Mandula, Phys. Rev. D30 (1984) 2230

[10] J. Engels, J. Fingberg, K. Redlich, H. Satz and M. Weber, Z. Phys. C42 (1989) 341

[11] M.N. Barber, in Phase transitions and critical phenomena, vol. 8, ed. C. Domb and J.L. Lebovitz (Academic Press, New York, 1983) p. 146

[12] M.E. Fisher, in Critical phenomena, Proc. of the 51st Enrico Fermi Summer School, Varenna, Italy, ed. M.S. Green (Academic Press, New York, 1971)

[13] G.A. Baker and J. Kincaid, J. Stat. Phys. 24 (1981) 469

[14] B.A. Freedman and G.A. Baker, J. Phys. A15 (1982) L715 